\title{
Investigación científica, práctica y experiencia: una reflexión sobre la formación de maestros en educación infantil
}

\author{
Scientific Research, Practice and Experience: \\ a Reflection about Preschool Teacher Training
}

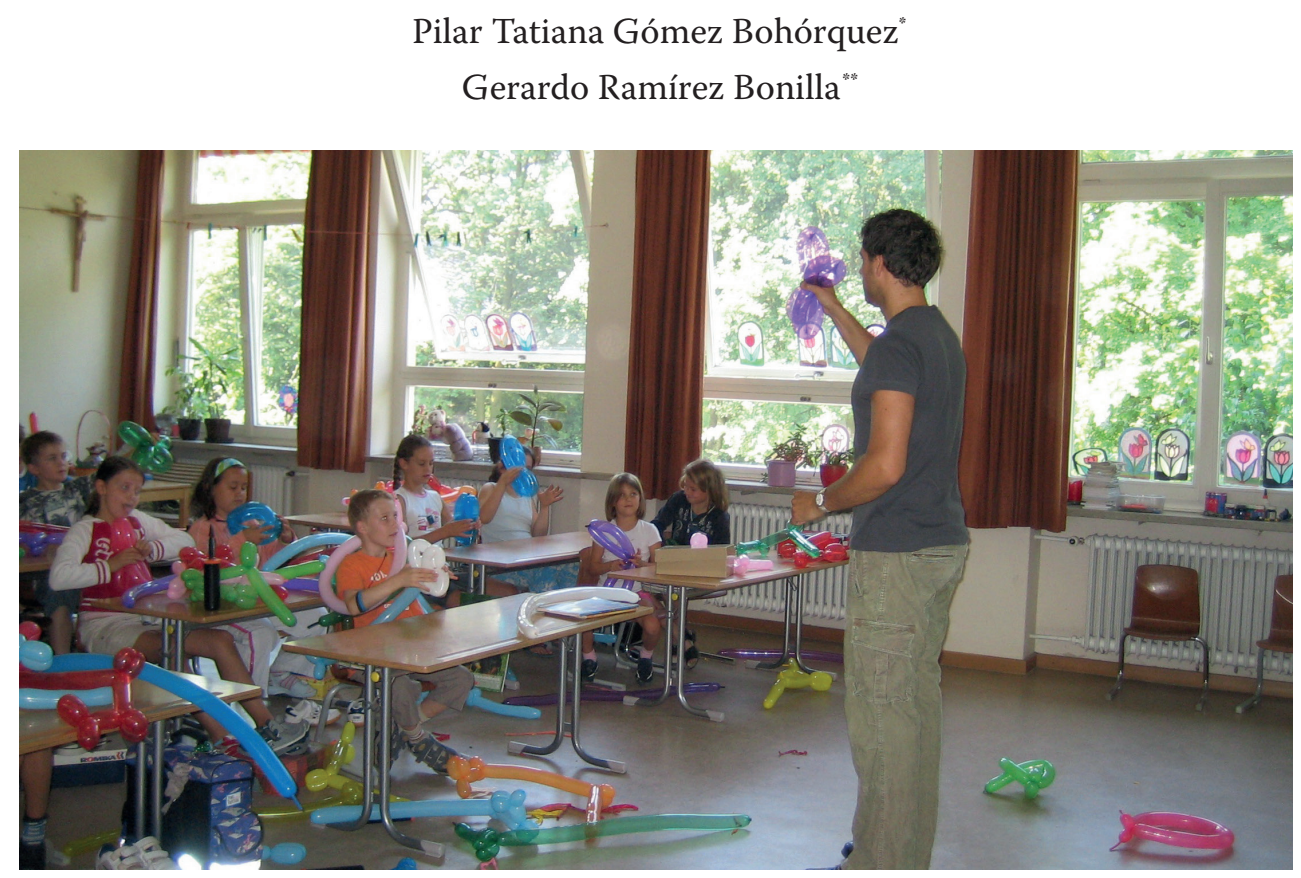

No hay práctica docente verdadera que no sea ella misma un ensayo estético y ético, valga la repetición.

(Freire, 2006, p. 46)

Citar este artículo como: Gómez Bohórquez, P. T. y Ramírez Bonilla, G. (2020). Investigación científica, práctica y experiencia: una reflexión sobre la formación de maestros en educación infantil. Revista Papeles, 12(23), 58-66.

Fecha de recibido: junio 30 de 2020. Fecha de aceptado: noviembre 10 de 2020.

* Licenciada en Educación preescolar, Magíster en docencia de la Universidad de la Salle, docente investigadora del programa Licenciatura en Educación Infantil de la Universidad de San Buenaventura, Bogotá. Miembro del grupo de Investigación Tendencias Actuales en Educación y Pedagogía (TAEPE). Experiencia laboral en educación superior en carreras profesionales como Lic. en educación Preescolar, Lic. en educación Básica, formando en asignaturas relacionadas con pedagogía, didáctica, evaluación e investigación. Correo electrónico: pgomez@ usbbog.edu.co

**: Licenciado en Filosofía, Especialista y Magíster en Filosofía Contemporánea. Docente investigador de la Universidad de San Buenaventura (Bogotá). Miembro del grupo de Investigación Tendencias Actuales en Educación y Pedagogía (TAEPE). Maestro orientador vinculado al programa de Licenciatura en Educación Infantil de la Universidad de San Buenaventura (Bogotá). Correo electrónico: gramirez@usbbog.edu.co 


\title{
Resumen
}

El documento aquí expuesto busca construir algunas reflexiones en el marco del conocimiento científico, la práctica formativa y las experiencias pedagógicas suscitadas en años de labor docente como formadores de maestros en educación infantil. Como primer elemento, se abordará la reflexión que el estudiante debe hacer de cara a su elección de formación profesional, entendiendo la autoevaluación como una estrategia continua de reconocimiento frente a su práctica (hacer), experiencia (ser) y saber pedagógico que construye desde la teoría y le da soporte a su quehacer (saber). En segunda instancia, se presentarán algunas reflexiones respecto a la relación existente entre la práctica formativa y la investigación científica vista desde una perspectiva formativa, las cuales deben ser parte constitutiva en el quehacer del educador infantil, pues es la única posibilidad de lograr transformaciones significativas en los contextos donde habitan los niños y las niñas. Finalmente, se esbozan algunas prospectivas con relación a lo que se debe considerar en el campo de la formación de maestros de manera que respondan a las situaciones emergentes y cambiantes que atañen a las infancias colombianas, para las cuales se debe estar preparado, pero no desde una visión idealista, sino de realidades que convocan, cuestionan y confrontan, a fin de generar procesos de reflexión.

Palabras clave: conocimiento científico, práctica formativa, educador infantil, reflexión, saber pedagógico, formación de maestros.

\begin{abstract}
This article aims at building some reflections framed by the scientific knowledge, and the authors' pedagogical experiences along all the years they had trained pre-service preschool teachers. The first element that will be presented is linked to the students' reflections about their professional choice. Thus, the students' self-evaluation is understood as a continuous strategy to recognize their practice (know how to do), experience (know how to be) and pedagogical knowledge, that the students construct based on theory to support their teaching practice (know how to know). The second element that will be presented is linked to reflections upon the relationship between pre-service practice and formative research. Those should be part of preschool teachers' practice since they offer a unique possibility to reach meaningful transformations in children's educational contexts. Finally, we suggest some forward planning related to what should be considered in the field of pre-service training in order to address emerging and changing situations that concern Colombian children. We consider that it is necessary to get ready for those situations not from an idealist point of view, but rather real situations trigger discussions, inquiries and confrontations that start reflection processes.
\end{abstract}

Keywords: scientific knowledge, pre-service training, preschool teacher, reflection, pedagogical knowledge, teacher training. 


\section{La reflexión: eje transversal en el quehacer del maestro}

La formación de maestros de educación inicial y su reconocimiento social ha sido una línea de investigación y de construcción permanente en los últimos años. Autores como Tello \& Verástegui (2016), Contreras \& Pérez (2010), Argos (2017), Gimeno (2013), Vaillant \& Marcerlo (2015) Flórez \& Vivas (2007) y Sandín (2003), entre otros, han enfocado sus análisis y discusiones a la dignificación de la labor, comprendiendo que esta se compone de diversas aristas que reflejan la relevancia del quehacer profesional del maestro de acuerdo con Sandín (2003):

La formación y el desarrollo profesional del docente ha dado lugar a la aparición de una serie de líneas de trabajo que abogan por la necesidad de analizar y comprender las vivencias, percepciones, sentimientos y conocimientos que los docentes activan cuando se enfrentan a las situaciones, singulares, inestables y ambiguas de su trabajo (p. 95).

En este sentido se reconoce, que la labor profesional de los maestros va más allá de un saber disciplinar o del cuidado asistencialista de los niños, que si bien es un elemento importante e inherente a la labor que realiza, no es la única capacidad con la que debe contar el educador

\section{La formación de maestros} invita a un constante proceso de reconstrucción del saber pedagógico ligado a rigurosos ejercicios de investigación científica sobre las prácticas, incentivando un repensar permanente del quehacer profesional. infantil; por el contrario, se reconocen como elementos fundamentales aquellas capacidades cognitivas, sociales, investigativas $y$ emocionales que permitirán un acercamiento más consciente y pertinente a las realidades propias de los estudiantes, para lograr generar experiencias y vínculos con quienes desarrollan su acción pedagógica.

En este sentido, la formación de maestros invita a un constante proceso de reconstrucción del saber pedagógico ligado a rigurosos ejercicios de investigación científica sobre las prácticas, incentivando un repensar permanente del quehacer profesional, y aunque los procesos de autoevaluación y reflexión no son una labor fácil, mucho menos cuando se está en formación, sí son tarea ineludible, pues como lo plantea Perrenoud (2004):

Formar a buenos principiantes es, precisamente, formar de entrada a gente capaz de evolucionar, de aprender con la experiencia, que sean capaces de reflexionar sobre lo que querían hacer, sobre lo que realmente han hecho y sobre el resultado de ello (p. 17).

Con relación a lo anterior, y partiendo de los procesos de reflexión e investigación que debe realizar permanentemente el educador infantil, se reconoce la reflexión como un elemento que permite comprender y reconstruir la realidad, la relación con el medio y los sujetos que hacen parte del quehacer profesional del maestro; reflexionar es activar y emplear de manera asertiva los procesos de pensamiento a fin de comprender los sucesos y realidades que se viven. La reflexión, en este sentido, es la base para la construcción del concepto de saber pedagógico y, ligado a este, al de la ética como un eje fundamental en la consolidación de un saber que es propio del maestro. Asimismo, la reflexión y la investigación posibilitan la comprensión de su rol en diversos escenarios donde se encuentra 
presente la infancia, buscando siempre generar aprendizajes significativos y perdurables.

Perrenoud (2004), citando a Schön, propone dos formas de ver la reflexión. La primera habla acerca de la reflexión en la acción o durante la acción, la cual lleva a preguntarse lo que pasa o va a pasar, lo que se puede hacer, muestra probables riesgos y oportunidades, brinda un abanico de posibilidades y herramientas con las que puede desarrollar acciones y experiencias pedagógicas. La segunda tiene que ver con la reflexión sobre la acción, en la que se consideran varios factores que inciden dentro de la práctica, como son: los objetivos planteados, los recursos, las acciones pedagógicas, los resultados y las evaluaciones. Lo mencionado en este último aspecto guarda estrecha relación con el papel de la investigación científica en el campo profesional del educador infantil, pues esta es la que le permite hacer innovaciones $\mathrm{y}$ transformaciones en su quehacer y en los contextos donde desempeña su labor.

La investigación es un asunto necesario si lo que se pretende es la cualificación permanente; es un proceso que no solo es complejo sino también riguroso y exigente, invita a repensar las maneras en que el maestro se relaciona consigo mismo, con el otro y con lo otro. Para ello, los educadores infantiles deben, desde su praxis, estar en constante reflexión, pues es la que posibilita la transformación de su práctica, tal como lo afirma Freire: "El momento fundamental en la formación permanente de los profesores es el de la reflexión crítica sobre la práctica. Es pensando críticamente la práctica de hoy o la de ayer como se puede mejorar la próxima" (Freire, 2004, p. 19). En línea con el pensamiento de Freire, se reconoce la reflexión como una práctica indispensable que le permite al educador infantil hacer una introspección de su labor con el único propósito de mejorar potencialmente su acción docente.

Dentro de la caracterización del educador infantil, se hace énfasis en la identidad profesional, la cual se entiende como "la percepción que los maestros tienen de sí mismos en cuanto al tipo de educadores que son, cómo se autodefinen y cómo les definen los demás el papel que les es reconocido en la sociedad" (Argos, 2017, p. 87). Es un proceso dinámico y continuo, de construcción y reconstrucción que inicia con la formación inicial y continúa durante la vida profesional del maestro, llevándolo a responderse cuestionamientos como ¿quién soy?, ¿hacia dónde voy? y ¿qué quiero llegar a ser? Cabe aclarar que definir la identidad profesional propia es una las principales dificultades que enfrenta cualquier persona al iniciar su vida laboral, debido a que en ciertas ocasiones no se tiene claridad frente a las motivaciones, capacidades y destrezas, lo que puede conducir a un ejercicio profesional sin sentido, mecánico y sin ninguna conexión afectiva.

\section{Práctica formativa e investigación científica}

Ahora bien, con el propósito de avanzar en las reflexiones que se vienen suscitando en torno a la formación de educadores infantiles, y como se ha venido mencionando, es importante analizar de qué manera los procesos de formación deben trascender para la consolidación de saberes y capacidades que permitan al maestro reconstruir su acción pedagógica, y para ello se propone trabajar en la triada de práctica, saber e investigación científica.
Los educadores infantiles a lo largo de su quehacer profesional confrontan un sin número de realidades y contextos, que les permiten afianzar diversas capacidades, entre estas: el trabajo en equipo, la construcción colectiva de saberes y las interacciones sociales; lo que les permite asumir un proceso de reconocimiento frente a sus propias fortalezas y debilidades, pues solo así se logrará descubrir, sentir y transformar la realidad 


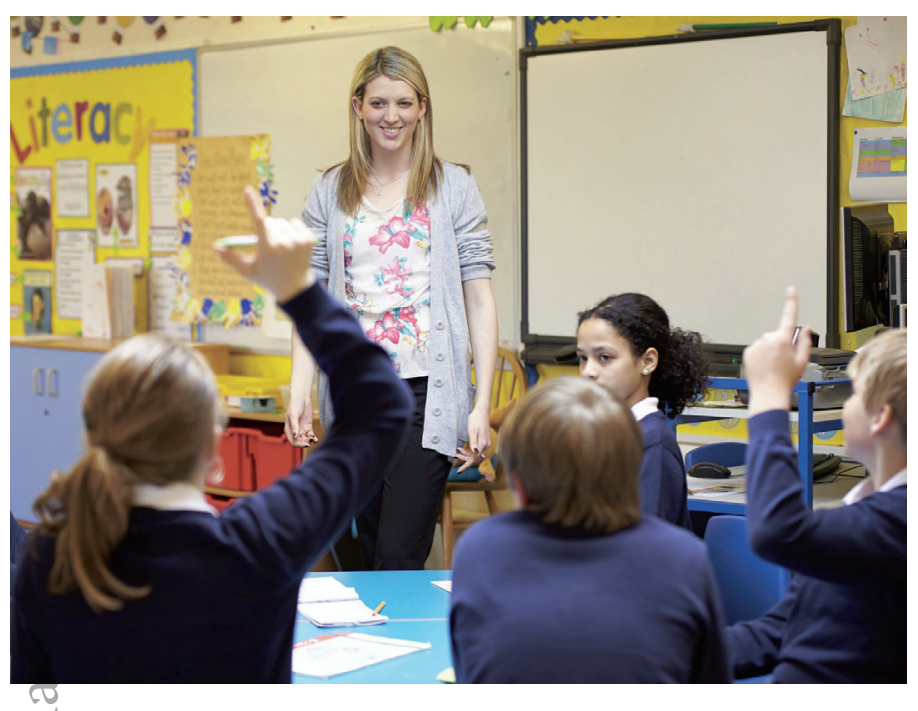

educativa, de allí la importancia de la autoevaluación como un acto inherente a la labor docente. En palabras de Flórez (1999):

La estrategia metacognitiva y autoreguladora a través de la cual el estudiante dirige con eficacia su aprendizaje es el primer y más importante objetivo de la enseñanza en cada área y constituye el procedimiento clave mediante el cual el alumno autoevalúa de forma permanente su progreso como aprendiz de pensador competente (p. 113).

Teniendo en cuenta lo anterior, el educador infantil que se encuentra en proceso de formación debe adquirir una serie de elementos que le posibilitarán afianzar su identidad profesional. En primer lugar, es importante el fortalecimiento de la dimensión cognitiva, la cual se entiende como aquella que le permite conocer, comprender y asimilar información conceptual respecto a su desarrollo profesional, consolidando capacidades de índole cognitivo como observar, analizar, inferir, sintetizar, entre otras, "[...] el profesor es, o debe ser, capaz de identificar los fenómenos educativos y distanciarse prudentemente de ellos, observándolos desde modelos de análisis adecuados para poderlos interpretar-explicar, generando así pertinentes teorías educativas y didácticas" (Cardona, 2013, p. 195).
Lo dicho, evidencia uno de los rasgos que deben caracterizar al educador infantil. Esto tiene que ver con la investigación científica, pues esta, al igual que en las ciencias positivas, parte de la observación de los fenómenos, el reconocimiento de variables e invariables, la implementación de diversos métodos y el uso de técnicas e instrumentos que le permitan hacer un desmonte crítico de la realidad social y educativa en aras de una transformación.

En segundo lugar, se encuentra la práctica, la cual no solo permite desenvolverse y poner en escena los conocimientos teóricos adquiridos en la academia, sino que también les abre la posibilidad de generar un entramado de reflexiones que, desde la acción, los lleva a construir y desarrollar su saber pedagógico articulando experiencias previas y sus vivencias en los procesos de enseñanza y aprendizaje. Es así como Cardona (2013) menciona:

[...] pues estos profesionales conocen, o deben conocer, cómo actuar educativamente, es decir, cómo potenciar a la persona y a la sociedad, cómo actuar para transmitir valores, cómo orientar en el camino del hombre hacia su plenitud. En definitiva, mediante su conocimiento pedagógico el docente sabe cómo modificar la realidad existente en base a un proyecto intencionado y en el marco de una opción moral concreta (p. 196).

En tercer lugar, se encuentra la investigación, la cual debe ser un elemento constitutivo en la formación docente, puesto que es a partir de ella que se lograrán cambios significativos en su ejercicio profesional y en los escenarios educativos en los cuales desarrolla su acción. Los profesionales de la educación deben comprender y poseer destrezas en investigación para resolver necesidades emergentes en la realidad educativa, partiendo de preguntas concretas que puedan ser sometidas a prueba, basados en una sólida metodología, en instrumentos y técnicas apropiadas, empleando un lenguaje práctico que llegue a todo nivel (McKernan, 2008) 


\section{Prospectivas sobre la formación de maestros}

Una vez analizados algunos elementos necesarios en la formación de educadores infantiles, es importante detenerse y repensar cuáles son aquellas prospectivas sobre la formación de maestros. Un primer punto de partida es el reconocimiento de la persona del estudiante, sus experiencias, conocimientos, intereses y motivaciones. Es necesario reconocer al estudiante desde el principio de realidad cultural, familiar y social, lo cual implica realizar un ejercicio de articulación de elementos en función de una acción formativa que responda a las necesidades del educando. Lo anterior ayudará a cristalizar la toma de decisiones y la asimilación de un proceso consciente que cumpla su finalidad, de modo que el estudiante asuma su proceso desde una perspectiva crítica, autocrítica, autónoma e integral.

Un segundo elemento que se debe considerar es la formación en capacidades sociales, y, para ello, primero será necesario comprender el concepto de capacidades. Nussbaum (2012) planeta que estas son semejantes a aquello que se conoce como "libertades sustanciales", y las entiende como "un conjunto de oportunidades (habitualmente interrelacionadas) para elegir y actuar" (p. 40), es decir, las capacidades le permitirán al educador reconocer sus mayores habilidades para tomar decisiones y desarrollar acciones de manera más oportuna y precisa. En este caso particular se hace referencia a las capacidades sociales y emocionales con las que debe contar el educador infantil, pues desde la emoción se generan apegos, relación estrecha y cercana que procuran el bienestar propio y del otro, logrando, de esta manera, una labor docente mucho más consiente y humanizada. En palabras de Nussbaum:

La forma fundamental de una emoción de fondo siempre es el amor o el apego a alguien considerado muy importante para el florecimiento propio, junto con alguna creencia general que signifique que el bienestar de esa persona no se encuentra plenamente bajo nuestro control (Nussbaum, 2008, p. 98).

Partiendo de los planteamientos de Nussbaum, las capacidades sociales y emocionales les permitirán a los educadores infantiles aprender a reconocerse como sujetos singulares y sociales, identificándose como seres únicos, pero también identificando a aquellos que los rodean como seres esenciales en su constitución personal y profesional; además, deben mostrar capacidad de respeto por los diversos ritmos y procesos de aprendizaje, no solo de sus estudiantes, sino de sus pares.

Es necesario que los maestros en formación reconozcan el compromiso con su proceso de construcción profesional, identificando que el trabajo en equipo implica asumir responsabilidades individuales y colectivas, que promuevan una labor mancomunada en pro de los procesos de enseñanza y aprendizaje. Lo anterior los invita a comprender todo lo que implica la labor docente, como lo plantea García (2010): "El objetivo prioritario de la educación debe ser capacitar a sus alumnos para el mayor desarrollo personal y ello requiere conocimientos, sentimientos y comportamientos responsables y solidarios" (p. 38). Desde esta perspectiva, el educador infantil debe promover la participación de todos los miembros de la comunidad, es decir, los niños, las familias, los maestros y demás agentes educativos, con el fin de entender y transformar las necesidades sociales en oportunidades para su desarrollo personal y el de la infancia. Asimismo, y bajo la premisa de aportar a la formación de capacidades sociales en el educador infantil, Vergara (2012) afirma que:

[...] el ejercicio de la ciudadanía, se funda en la responsabilidad de saberse interpelado por el otro, con la idea de 
deliberación y argumentación racional como principios de consenso, el cual cobra sentido si existe una ciudadanía responsable donde se privilegia la ética, como un conocimiento que promueve que los sujetos desarrollen procesos de socialización y participación que les permita expresar sus propias opiniones, y construir argumentos relativos a las condiciones en que a cada uno le corresponde orientar su propia vida (18).

En línea con lo anterior, es importante señalar que la profesión docente no se basa únicamente en la construcción de maestros sabios desde las teorías estudiadas y fundadas o expertos en investigación social o educativa, sino que, además, requiere de una persona equilibrada emocionalmente, pues la tarea formativa se da a partir de un acompañamiento permanente de niños que se encuentran en crecimiento y en construcción de su personalidad, los cuales en muchas ocasiones presentan vacíos afectivos, necesidades educativas y problemas de comportamiento. Nussbaum (2012), refiriéndose a las diez capacidades que debería desarrollar el ciudadano, considera que las emociones juegan un papel fundamental en la constitución del sujeto y lo plantea en los siguientes términos:

Poder sentir apego por cosas y personas externas a nosotras y nosotros mismos; poder amar a quienes nos aman y se preocupan por nosotros, y sentir duelo por su ausencia; en general, poder amar, apenarse, sentir añoranza, gratitud e indignación justificada. Que no se malogre nuestro desarrollo emocional por culpa del miedo y la ansiedad (p. 55).

Es por ello que un tercer elemento a considerar en la formación de educadores infantiles es la inteligencia emocional, la cual les permitirá desarrollar capacidades para autorregularse en momentos de crisis, sopesando las distintas variables que deben tener en cuenta al momento de proceder o tomar cualquier tipo de decisión. De igual manera, la inteligencia emocional es fundamental para entablar relaciones asertivas basadas en el respeto, la tolerancia, la equidad y la solidaridad. Como lo afirma Darder (2012):

[...] las emociones forman parte esencial de los humanos y afirman la unidad de la persona. Prescindir de las emociones es una simplificación que mutila la complejidad de la realidad y de la educación. Las vivencias emocionales y afectivas del sujeto intervienen y condicionan su desarrollo personal y, por lo tanto, el aprendizaje (p. 16).

Como cuarto elemento a tener en cuenta es la reflexión y la investigación continua frente al hacer, el saber y el ser, pues no se trata únicamente de erudición, sino de aportar a la construcción de sujetos críticos e investigativos, capaces de evolucionar y revolucionar la educación, a partir del estudio minuciosos de problemáticas sociales, culturales, políticas $\mathrm{y}$ educativas, construyendo capacidades $\mathrm{y}$ saberes fundamentados en la acción y en la experiencia pedagógica. Así lo afirma Torres (2018):

La formación de educadores capaces de enfrentar los retos ineludibles e inaplazables de la sociedad actual reclama nuevos modelos acordes con el contexto cultural en los aspectos socioeconómicos, políticos y científico-tecnológicos determinantes del tercer milenio, de manera que se garantice una profesionalización con calidad ética y pedagógica en las diferentes áreas, saberes y disciplinas (p. 124).

En quinto lugar, aquellos que se dedican a la tarea educativa deben tener fundamentos sólidos en investigación científica y social, pues es la que les permitirá hacer transformaciones en su propia práctica y en los modos habituales en los que se desarrolla el ejercicio de la docencia. Torres (2018) afirma que: 
En la sociedad del conocimiento la calidad de la educación superior está íntimamente asociada con la práctica de la investigación, práctica que se manifiesta de dos maneras: enseñar a investigar y hacer investigación. La primera hace alusión al ejercicio de la docencia investigativa, esto es, a utilizar la investigación en la docencia, tanto para darle pertinencia científica a esta, como para familiarizar a los estudiantes con la lógica de la investigación e iniciarlos en su práctica, es decir para adelantar formación investigativa. La segunda hace alusión a la producción o generación sistemática de conocimiento y a su aplicación para resolver problemas del contexto (p. 189).

Todo lo dicho hasta ahora, permite concluir que un educador infantil debe desarrollar una serie de capacidades sociales, emocionales, cognitivas e investigativas que le permitan realizar su acción pedagógica en cualquier contexto donde habite la infancia, teniendo en cuenta que "las competencias no se adquieren en una etapa de formación inicial y se aplican sin más, sino que se crean y se recrean continuamente en la práctica profesional" (Pavié 2011, p. 78). Es así como se puede reiterar que el educador infantil debe encontrar en la reflexión su mejor aliada, articulándola a procesos investigativos y de introspección, logrando desarrollar capacidades de pensamientos crítico, enmarcadas la formación de educadores infantiles implica la comprensión del educando como un sujeto afectivo, histórico, social y consciente de la cultura que vive, se construye y reconstruye a partir de la observación reflexiva y crítica de su realidad; su quehacer implica un vínculo estrecho entre la teoría y la práctica.

también desde su ética profesional, lo que le permitirá relacionarse con los otros y con lo otro de la manera más diáfana posible.

Finalmente, es importante reconocer que la formación de educadores infantiles implica la comprensión del educando como un sujeto afectivo, histórico, social y consciente de la cultura que vive, se construye y reconstruye a partir de la observación reflexiva y crítica de su realidad; su quehacer implica un vínculo estrecho entre la teoría y la práctica, generando nuevos conocimientos a partir de la investigación. Es así como se le reconoce como un agente social capaz de generar reflexiones, tomar decisiones asertivas, convirtiéndose en sujeto de cambio e innovación en el campo educativo y social.

\section{Referencias}

Argos, J. (2017) Fundamentos teóricos de la educación infantil. Santander, España: Ediciones Universidad de Cantabria.

Barrientos, L (10 Agosto 2011) Democracia representativa y conflicto social. [En línea] Recuperado el 02 de abril de 2020 en: http:// www.revistafusion.com/201108102203/ Opinion/Opinion/democracia-representativa-y-conflicto-social.htm
Cardona, J. (2013). Epistemología del saber docente. Madrid, España: Universidad Nacional de Educación a Distancia.

Contreras, J \& Pérez, N. (2010) Investigar la experiencia educativa. Madrid, España: Ediciones Morata S.L.

Darder, P. (2012). Aprender y educar con bienestar y empatía. La formación emocional del profesorado. Barcelona, España: Ediciones OCTAEDRO, S.L 
Flórez, R. \& Vivas, M. (2007) La formación como principio y fin de la acción pedagógica. Revista educación y pedagogía, volumen XIX, núm. 47, enero-abril. [En línea] Recuperado el 18 enero 2021 en: https:// revistas.udea.edu.co/index.php/revistaeyp/ article/view/6680/6122\}

Flórez, R. (1999). Evaluación, Pedagogía y Cognición. Bogotá: Colombia. Editorial McGraw-Hill. P. 113.

Freire, P. (2006) Pedagogía de la autonomía: Enseñar exige reflexión crítica sobre la práctica. [En línea] recuperado mayo 27 de 2016. http://www.mercaba.org/ ARTICULOS/P/pedagogia_de_la autonomia_freire.htm

García, E. (2010). Competencias éticas del profesor y calidad de la educación. Universidad Complutense. [En línea] recuperado mayo 27 de 2016. http://www.revistafusion. com/201108102203/Opinion/Opinion/ democracia-representativa-y-conflictosocial.htm

Gimeno, J. (2013) En busca del sentido de la educación. Madrid, España: Editor: Ediciones Morata S.L.

McKernan, J. (2008). Investigación - acción y curriculum. Madrid, España: Ediciones Morata.

Nussbaum, M. (2008). Paisajes del pensamiento: la inteligencia de las emociones. Barcelona, España: Paidós.

Nussbaum (2012). Crear capacidades. Propuesta para el desarrollo humano. Barcelona, España: Paidós.

Pavié, A. (2011). Formación docente: hacia una definición del concepto de competencia profesional docente. Vol. 14, No. 1 REIFOP. 14 (1). 67-80. [En línea] recuperado 18 enero de 2021. https://dialnet.unirioja.es/ servlet/articulo? codigo $=3678767$

Perrenoud, P. (2004). Desarrollar la práctica reflexiva en el oficio de enseñar. Profesionalización y razón pedagógica. México D.F. Graó

Sandín, M.E. (2003). Investigación Cualitativa en Educación Fundamentos y Tradiciones. Editorial McGraw Hill / Interamericana de España S.A.U Madrid.

Tardif, M. (2004). Los saberes del docente y su desarrollo profesional. Narcea ediciones. Madrid, España.

Tello, Y \& Verástegui, E. (2016). El saber y el hacer de la investigación acción pedagógica. Cusco Peru: Inversiones Dalagraphic. [En línea] recuperado 18 enero de 2021. http://repositorio.uncp.edu.pe/bitstream/ handle/UNCP/1192/libro\%20IAP\%20 de\%20junio\%20de\%202016-LISTOcc. pdf? sequence $=1$ \&isAllowed $=\mathrm{y}$

Torres, F (2018). La formación de educadores: Una mirada cultural. Caldas: Ediciones Universidad de Caldas.

Vaillant, D \& Marcerlo, C. (2015) El ABC y D de la formación docente. Madrid: NARCE S.A. Ediciones.

Vergara, M. (2012) El conocimiento de la educadora infantil: una aproximación a su construcción. Revista Papeles - Volumen 4 No. 8 • pp. 8-22 • Julio - diciembre de 2012. [En línea] recuperado 18 enero de 2021. http://revistas.uan.edu.co/index.php/ papeles/article/view/224/190 